\title{
Histological Characteristics of the Skin and Cuticle of the Wool Fibers in Dubska Pramenka and the Use in
}

\section{Industry}

\author{
Nadzida Mlaco ${ }^{1}$, Ervin Bucan ${ }^{2}$, Amela Katica ${ }^{1}$, Almira Softic ${ }^{3}$, Velija Katica ${ }^{3}$, Vedad Sakić ${ }^{3}$, Selma Pilic ${ }^{4}$ and \\ Jasmin Katica ${ }^{5}$ \\ 1. Department of Anatomy and Histology with Embryology, Veterinary Faculty, University of Sarajevo, Sarajevo 90,71000, Bosnia \\ and Herzegovina \\ 2. Directorate for Food Safety, Veterinary and Phytosanitary Affairs, Podgorica 26,81000, Montenegro \\ 3. Department of Animal Science, Veterinary Faculty, University of Sarajevo, Sarajevo 90,71000, Bosnia and Herzegovina \\ 4. Department of Biology, Faculty of Mathematics and Natural Sciences, University of Sarajevo, Sarajevo 33-35, 71000, Bosnia and \\ Herzegovina \\ 5. Department of Feed and Nutrition of Domestic Animals, Veterinary Faculty, University of Sarajevo, Sarajevo 90, 71000, Bosnia \\ and Herzegovina
}

\begin{abstract}
Microscopic analysis of the skin and cuticle of wool fibers in Dubska pramenka show different qualitative parameters. The research study included different parts of the body and the samples of the wool fibers from different regions of the body. Histological description shows difference in the basic structurers of the skin in Dubska pramenka. A very pronounced thin epidermis, while dermis and hypodermis are more developed on the samples from the shoulder. Cornified flakes-cuticle of the root of the tail was of a fine structure, and cornified flakes from the rump showed the features of the rough wool fibers. "Transitional form of cuticle" was dominant on the shoulder. The qualitative parameters have a significant influence on the overall quality of wool in general, and the research contributes to a greater usability value of the wool and development of livestock farming in areas in a broader sense.
\end{abstract}

Key words: skin, cuticle, Dubska pramenka.

\section{Introduction}

Skin is the largest organ and it covers the entire body. It consists of epidermis, dermis and subcutaneous tissue or subcutis. The structure of skin in domestic animals is not equal, primarily, the epidermis-dermis ratio varies, the presence and thickness of subcutaneous connective tissue. Difference in histological structure of some areas of the body is also present in the same animals [1]. Wool, which is a product of the skin, is made of a set of wool fibers. The surface of the wool fibers contains cornified cells, flakes, i.e. cuticle. Katica, A., et al. [2]

Corresponding author: Nadzida Mlaco, Ph.D., main research field: histology in veterinary medicine. studies that focused on the structure of the skin and the characteristics of the cuticle found differences in the structure, especially in the outer layer of the skin-epidermis, and the appearance of the cuticle, which varied depending on the region of the body. In general, total quality of the wool depends on the qualitative parameters of the skin and fibers and so does its classification and theuse in the processing and development of livestock farming in areas.

\section{Material and Methods}

The samples for microscopic examinations of the skin and fibers of Dubska pramenka were taken from Vlasic mountain in Central Bosnia. The animals were reared under nomadic conditions, at the latitude of 
$850-1,600 \mathrm{~m}$. The animals were healthy with a good body condition, weighing between 50 and $65 \mathrm{~kg}$, above three years of age. Nutrition of the sheep was usual for nomadic conditions during summer, with the addition of grains before estrus, in line with the research studies [3,4]. The samples of the skin for histological analysis were taken from the middle of the last left rib, the shoulder, the rump and the root of the tail according to the Markotic method. A moment before sampling, the site was previously shaved, washed and disinfected, and skin cuts with subcutis were prepared, $6 \mathrm{~mm}$ in diameter. The samples were placed in plastic bottles with caps that contained $10 \%$ formalin until histological processing i.e. molding into paraffin blocks. Histological preparations of the skin were prepared according to the usual sample preparation protocol for optical microscopy observations. The analysis of the microscopic skin preparations was performed using binocular optical microscope MOTIC TYPE $120 \mathrm{M}$ with magnification of 100, 200 and 400 times. The fiber for microscopic analysis was taken from different parts of the body: the shoulder, the rump and the root of the tail, cutting the wisps next to the skin. Prior to microscopic examination of the cuticle, all mechanical dirt is cleaned; the samples were washed with neutral soap and rinsed with water, and then rinsed with distilled water. After that, the samples were immersed in hydrogen peroxide for 24 hours, then in xylol for 48 hours. These treated samples were rinsed with distilled water and left to dry. The processed samples were placed on the glass slide, embedded into glycerin and examined under the microscope. The fibers were analyzed with binocular optical microscope, with magnification of 100, 200 and 400 times.

\section{Results and Discussion}

Microscopic analysis of the Dubska pramenka skin was performed using epidermis, dermis and hypodermis. Using descriptive presentation, the particulars of the skin of Dubska pramenka were singled out from different parts of the body. Microscopic examination of the skin of the Dubska pramenka taken from the shoulder identified a very thin epidermis, with dermis and hypodermis well developed. The attention was focused on dermis and in that particular segment, that the presence of hair follicles grouped in sets of two or three follicles (one central and two peripheral) and rarely asingle follicle was found [5]. Reticular dermis contains collagen and elastic fibers that give dermis the appearance of compactness (Fig. 1). Sebaceous glands (Glandulae alveolares) are immediately next to hair follicles. Sweat glands are surrounded by the elements of connective tissue where adipocytes can be seen [6].

The analysis of the sample fibers from the shoulders shows a so called transitional form of cuticle (Fig. 2). The cornified flakes are rather large with multi-corners, and the clearly pronounced edges are merging forming a whole.

A thin epidermis is characteristic on the rump of Dubska pramenka. Dermis is well developed, with connective tissues and notable nuclei of the smooth muscle cells. The compacted collagen and elastic fibers are present in the papillary layer, while hair follicles are surrounded with poorly developed sebaceous glands, which are globular or elongated (Fig. 3).

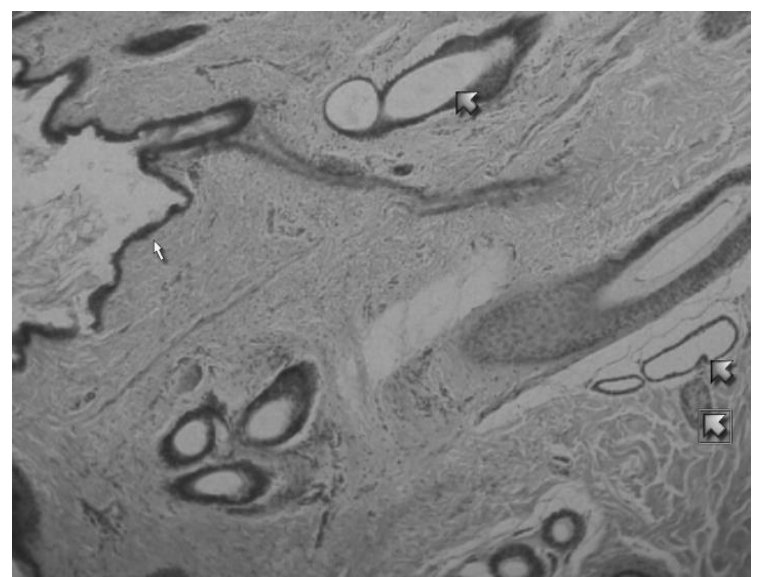

Fig. 1 The skin of the shoulder (Dubska pramenka); the white arrow indicates dermis; the blue arrows indicate sweat glands; the green arrow indicates sebaceous gland; HXE, $\times 200$. 

Pramenka and the Use in Industry

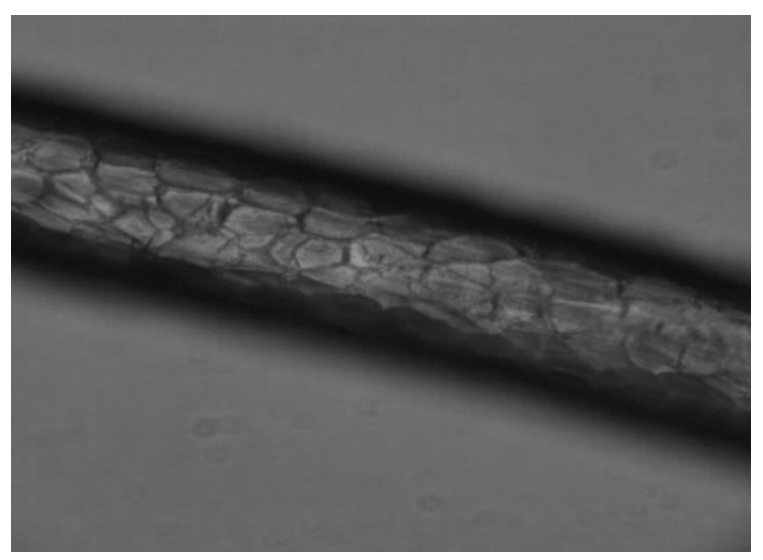

Fig. 2 Native preparation; $\times$ 400; shoulder.

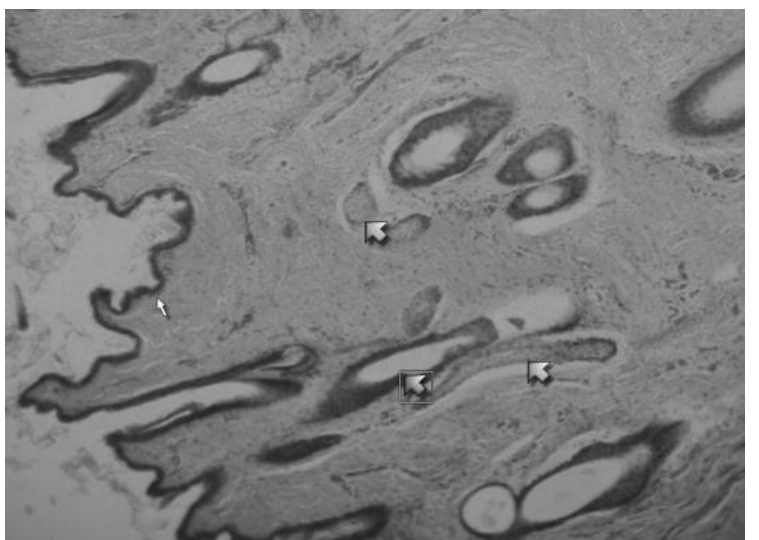

Fig. 3 The skin of the rump (Dubska pramenka); the white arrow indicates epidermis; the blue arrow indicates hair follicle; the green arrow indicates sebaceous gland; HXE; $\times 200$.

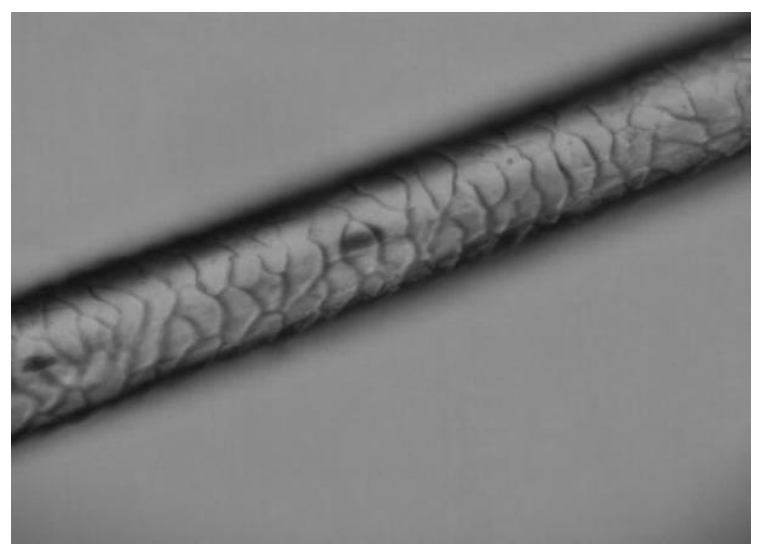

Fig. 4 Native preparation; $\times 400$; the rumps.

On the wool fiber samples taken from the rump, cornified flakes overlap continuously (Fig. 4). They have irregular shape reminding of roof tiles. This appearance of the cuticle most frequently is associated with rough wool fibers.
Epidermis of the root of the tail is notable, but without clear strata. Its surface shows a layer of squamous cells, darker in color, flat, interconnected. This layer makes the stratum corneum, made of cells without nuclei, with cytoplasmic inclusions of keratin. The skin of the root of the tail shows clearly visible outer cells separated from other layers that do not have clear edges, and they make stratum disjunctum. Dermis is rich with oval hair follicles with developed sebaceous glands. Loose connective tissue is present in the outer papillary layer, while deeper dermis layers also contain collagen fibers (Fig. 5).

Cross sections of sebaceous glands are noted (Glandulae glomiformes), which is highlighted in the studies by Janquera L. C. [7]. The studies to date indicate that the distribution of sebaceous glands in this part of the skin may affect the quality of leather, leather industry, as it leads to separation of the papillary section from the reticular section of the dermis, which affects the quality of the final products in leather industry.

Cuticle i.e. flakes on the fibers from the root of the tail are significantly smaller, of finer structure, (Fig. 6), especially in regard to the ones from the rump.

Infundibular appearance of circularly arranged cornified flakes may be seen on the edges of the wool fibers (Fig. 7).

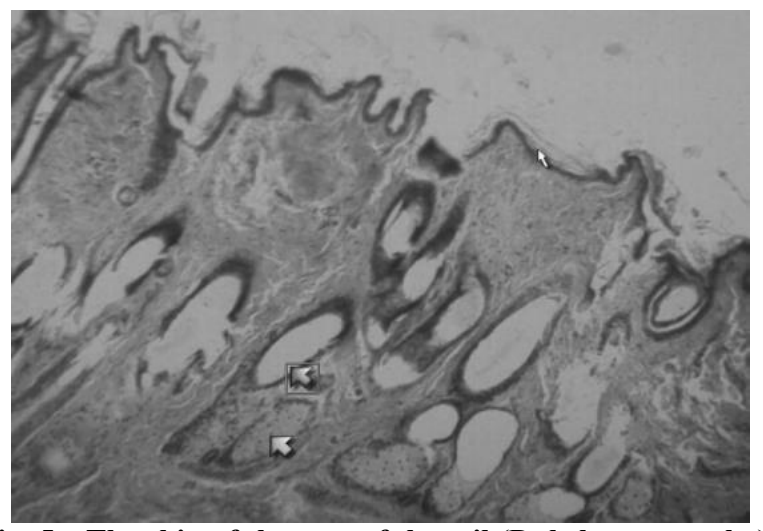

Fig. 5 The skin of the root of the tail (Dubska pramenka); the white arrow indicates dermis; the blue arrow indicates hair follicle; the green arrow indicates sebaceous gland; HXE; $\times 200$. 


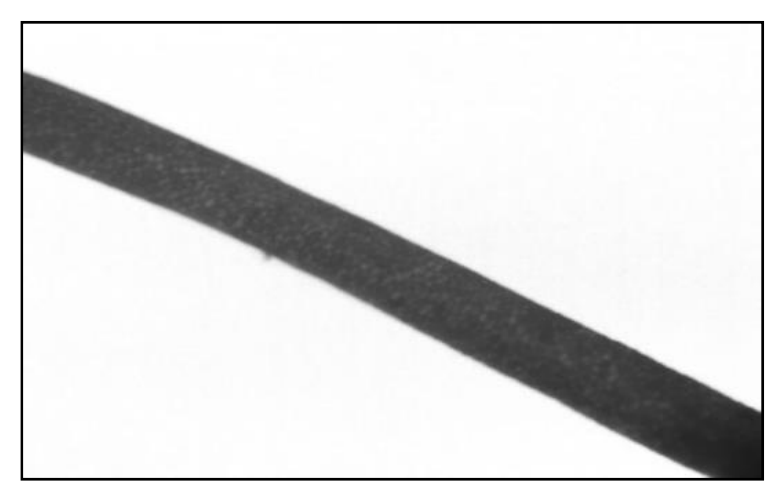

Fig. 6 Native preparation; $\times 400$; the root of the tail.

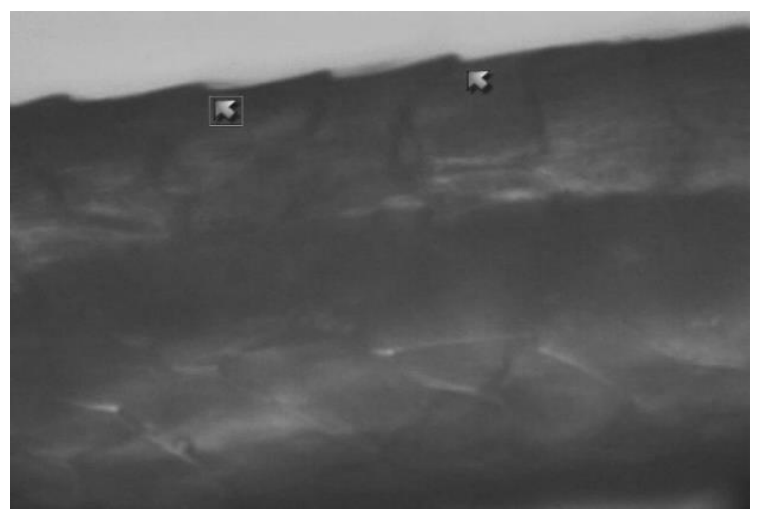

Fig. 7 Native preparation; immersion; distribution of flakes; the root of the tail.

This appearance is a characteristic of a finer high-quality wool, which contributes to high-quality classification bearing in mind the quality of the final products of the textile industry.

\section{Conclusions}

Description of histological preparations of the Dubska pramenka skin showed the following distinctive features: thin epidermis with developed dermis and hypodermis - the shoulder samples. Compacted collagen and elastic fibers are present in the papillary layer of dermis of the skin from the rump, stratum corneum and stratum disjunctum of epidermis of the skin from the root of the tail are clearly visible unlike in other layers. The studied samples of the wool fibers show certain differences in the form and position of the cornified flakes-cuticle depending on the region of the body. The cuticle of the root of the tail was of a finer structure; cornified flakes on the rump showed the features of rough wool fibers, and the "transitional form of the cuticle" was dominant on the shoulder. Distinctive features of the skin structure and appearance of the cuticle of the wool fibers of Dubska pramenka may be used as comparative advantages in the utilization of new raw materials in different industry branches and may directly influence improvement of production in livestock farming. Considering that skin has multiple functions in an organism and together with cuticle, it accentuates its protective role, it directs the further use of wool as an important ecological raw material.

\section{Acknowledgement}

The study is likely to have an impact on total production and use of raw wool as the main raw material in the demanding textile industry, which from an environmental point of view, is a major problem both in Bosnia and Herzegovina, as well as in neighboring countries.

\section{References}

[1] Kozaric, Z. 1997. Veterinary Histology. Zagreb: Veterinarska izdanja, Naklada Karolina.

[2] Katica, A., Mlaco, N., Sakic, V., Satrovic, E., and Kovcic, I. 2015. Skin and Derivate of Skin of Animals. Veterinary Faculty, University of Sarajevo.

[3] Palian, B. 1956."About Seasonal Changes in Wool Fibres of Pramenka on the Kupres." Veterinaria 5: 3-11.

[4] Smalcelj, I. 1951."Does the Season Affect the Fineness of the Wool in Pramenka Sheep." Godisnjak 3: 225-32.

[5] Vegara, M. 1991."Development of Wool Follicles of Pramenka Sheeps in the Age of Six to Twenty-Six Months." Doctoral Thesis, University of Sarajevo.

[6] Katica, A., Mlaco, N., Hasanbasic, D., and Hamzic, E. 2010. Basic of Veterinary Histology. Sarajevo: Veterinary Faculty, University of Sarajevo.

[7] Junqueira, L. C., and Carneiro, J. 2005. Basic of Histology, Text and Atlas, Data status, Eleven Edition. Novi Sad. 